\title{
ANALYTICITY IN THE BOUNDARY OF A PSEUDOCONVEX DOMAIN
}

\author{
ALAN V. NOELL
}

\begin{abstract}
Let $D$ be a bounded pseudoconvex domain with $C^{\infty}$ boundary in $\mathbf{C}^{n}, A^{\infty}(D)$ the algebra of functions holomorphic in $D$ and $C^{\infty}$ up to the boundary, and $M$ a compact real-analytic manifold in the boundary which is integral for the complex structure of the boundary and which has no complex tangent vectors. A necessary and sufficient condition that each element of $A^{\infty}(D)$ be real-analytic on $M$ is that the germ of the complexification of $M$ be in the boundary. Examples indicate that the quasi-analyticity of $A^{\infty}(D)$ along $M$ is possible even in the absence of complex manifolds in the boundary.
\end{abstract}

1. Introduction. We call a smooth manifold $M$ in the boundary of a domain an integral manifold if its tangent space at each point is contained in the maximal complex subspace of the tangent space of the boundary. $M$ is totally real if it has no complex tangent vectors; more precisely, if $J$ is the almost complex structure, the condition is that $T_{p}(M) \cap J T_{p}(M)=0$ for all $p \in M$. A well-known theorem due to Stein states that holomorphic functions which are Lipschitz on $\bar{D}$ are twice as smooth when restricted to integral curves. (For the precise statement we refer the reader to $[9$, Corollary 2, p. 443].) In this note we investigate what conditions on $D($ or $\partial D)$ imply high regularity of functions in $A^{\infty}(D) \mid M=\left\{f \mid M ; f \in A^{\infty}(D)\right\}$; here $M$ is a compact totally real real-analytic integral manifold in $\partial D$. Our results depend on the notion of a complexification of such a manifold. Suppose $M$ has real dimension $m$. Locally (near $p \in M$ ) we take a real-analytic parametrization $\phi: V \rightarrow M$, where $V$ is a neighborhood of 0 in $\mathbf{R}^{m}$ and $\phi(0)=p$. The holomorphic extension $\Phi$ of $\phi$ to a neighborhood $V^{\prime}$ of 0 in $\mathbf{C}^{m}$ is nonsingular since $M$ is totally real; then $\Phi\left(V^{\prime}\right)$ is a complexification of $M$ near $p$. Using the compactness of $M$ we combine these to get a complex submanifold $M^{\prime}$ of a neighborhood $W$ of $M$ which has complex dimension $m$ and which contains $M$ as a submanifold. Details of this construction are in $[\mathbf{1 0}$, p. 1274]. Note that, assuming the connectedness of $M^{\prime} \cap W$, for each real-analytic function on $M$ there are a neighborhood $W^{\prime}$ of $M$ and a unique extension of the function to $H\left(W^{\prime} \cap M^{\prime}\right)$. (Here, as elsewhere, $H(N)$ denotes the algebra of holomorphic functions on the (connected) complex manifold $N$.) Our main result can then be stated as follows.

THEOREM. Let $D$ be a bounded pseudoconvex domain in $\mathbf{C}^{n}$ with $C^{\infty}$ boundary, $M$ a compact totally real real-analytic integral manifold in $\partial D$, and $M^{\prime}$ a complexification of $M$ in $W$. Then each element of $A^{\infty}(D) \mid M$ is real-analytic if and only if there is a neighborhood $U \subseteq W$ of $M$ so that $U \cap M^{\prime} \subseteq \partial D$.

Received by the editors July 5,1984 .

1980 Mathematics Subject Classification. Primary 32A40; Secondary 32E25.

Key words and phrases. Pseudoconvex domain, integral manifold, complexification. 
The proof of this theorem is in $\S 2$. We remark that obviously pseudoconvexity is required in the theorem; furthermore, some minimal smoothness of the boundary is necessary. In fact, Sibony constructed in [8, p. 973] a bounded pseudoconvex domain in $\mathbf{C}^{2}$ (with nonsmooth boundary) so that all bounded holomorphic functions on the domain extend to be holomorphic on a strictly larger domain.

Motivation for this work came from a study of interpolation in [6]; there an example is given of a class of domains for which $A^{\infty}(D)$ gains a good deal of smoothness upon restriction to an integral curve. In $\S 3$ we further discuss this example as a contrast to the theorem above. In particular, we give the following

EXAMPLE. There exists a convex domain $D \Subset \mathbf{C}^{2}$ which is strongly pseudoconvex off of a line segment $K$ so that $A^{\infty}(D)$ is quasi-analytic along a subinterval of $K$.

Our proof of the theorem depends on the identification of the spectrum of the algebra $A^{\infty}$ given by Hakim and Sibony in [3, Theorem 1, p. 128]. Recall that $A^{\infty}(D)$ is a Fréchet algebra with the family of norms given by

$$
P_{N}(f)=\sum_{|\alpha| \leq N} \frac{1}{\alpha !}\left\|D^{\alpha} f\right\|_{\bar{D}}
$$

here, as elsewhere in this note, $\|g\|_{X}$ denotes the supremum of $|g|$ on $X$.

THEOREM (HAKIM-SIBONY). If $D$ is a bounded pseudoconvex domain with $C^{\infty}$ boundary, then the space of nonzero continuous complex homomorphisms of $A^{\infty}(D)$ can be identified with $\bar{D}$.

2. Proof of the theorem. Suppose that, for some neighborhood $U$ of $M$, $U \cap M^{\prime} \subseteq \partial D$. If $f \in A^{\infty}(D)$, then $\bar{\partial} f \equiv 0$ in $\bar{D}$, so $f$ is holomorphic on $U \cap M^{\prime}$. It follows that $f$ is real-analytic on $M$. Thus each element of $A^{\infty}(D) \mid M$ is realanalytic.

For the nontrivial part of the proof, we assume each element of $A^{\infty}(D) \mid M$ is realanalytic and fix a point $p \in M$. For each $f \in A^{\infty}(D) \mid M$ there is a neighborhood $V$ of $p$ (depending on $f$ ) so that $f$ extends to be holomorphic on $V \cap M^{\prime}$. Our first step is to remove the apparent dependence of $V$ on $f$ (cf. the argument in $[3, \mathrm{p}$. 131]). Let $B(r)$ denote the open ball with center $p$ and radius $r>0$; let $X(r)$ be the Fréchet space of pairs $(F, f)$ with $F \in H\left(B(r) \cap M^{\prime}\right), f \in A^{\infty}(D)$, and $F=f$ on $B(r) \cap M$; and, let $\rho(r): X(r) \rightarrow A^{\infty}(D)$ be the restriction map. We know that the union of the images of $\rho(r)$ over $1 / r=1,2,3, \ldots$ is $A^{\infty}(D)$, so, for some $r_{1}$, the image of $\rho\left(r_{1}\right)$ is of the second category in $A^{\infty}(D)$. By the open mapping theorem for Fréchet spaces (e.g., [7, p. 47]), $\rho\left(r_{1}\right)$ is surjective. Thus, if $V=B\left(r_{1}\right)$, each element of $A^{\infty}(D) \mid M$ extends to be holomorphic on $V \cap M^{\prime}$.

The second step is to show that $V \cap M^{\prime} \subseteq \bar{D}$. Fix a point $q \in V \cap M^{\prime}$ and define a complex homomorphism $\chi: A^{\infty}(D) \rightarrow \mathbf{C}$ by $\chi(f):=F(q)$ if $f \in A^{\infty}(D)$ and $F$ is an extension of $f$ which is holomorphic on $V \cap M^{\prime}$. Since the extension is unique, $\chi$ is well defined, and the following argument shows that $\chi$ is continuous: If $g \in A^{\infty}(D)$, then $|\chi(g)| \leq\|g\| \bar{D}$, for otherwise $g-\chi(g)$ would be invertible in $A^{\infty}(D)$, an impossibility. Thus, if $f_{j} \rightarrow f$ in $A^{\infty}(D)$, from $\left\|f_{j}-f\right\|_{D} \rightarrow 0$ it follows that $\chi\left(f_{j}\right) \rightarrow \chi(f)$. Hence, $\chi$ is continuous. By the aforementioned result of Hakim and Sibony, $\chi$ is given by evaluation at a point of $\bar{D}$, and it is clear that this point must be $q$. If follows that $q \in \bar{D}$ and so $V \cap M^{\prime} \subseteq \bar{D}$. 
The third step is to show that, in fact, $V \cap M^{\prime} \subseteq \partial D$. For this we use the fact that there is a function $\sigma \in C(\bar{D})$ which is plurisubharmonic on $D$ and satisfies $\sigma<0$ on $D$ while $\sigma=0$ on $\partial D$; this is a simple form of the theorem of Diederich and Fornaess [2, Theorem 1, p. 131] on bounded plurisubharmonic exhaustion functions. We claim that $\sigma$ is actually plurisubharmonic on $V \cap M^{\prime}$. To see this, fix $q \in V \cap M^{\prime}$, let $\mathbf{n}$ be the outward unit normal to $\partial D$ at $q$, and let $V^{\prime} \subset V$ be a small neighborhood of $q$. Since $V \cap M^{\prime} \subseteq \bar{D}$, if $\varepsilon>0$ is small, then

$$
\left\{t-\varepsilon \mathbf{n} ; t \in V^{\prime} \cap M^{\prime}\right\} \subseteq D .
$$

Thus $\sigma(t)$ is the uniform limit on $V^{\prime} \cap M^{\prime}$ of the plurisubharmonic functions $\sigma_{\varepsilon}(t):=$ $\sigma(t-\varepsilon \mathbf{n})$ as $\varepsilon \rightarrow 0$; it follows that $\sigma$ is plurisubharmonic on $V^{\prime} \cap M^{\prime}$. Since $q$ was arbitrary, $\sigma$ is plurisubharmonic on $V \cap M^{\prime}$, giving the claim. Now $\sigma$ attains its maximum value at the (relative) interior point $p$ of $V \cap M^{\prime}$; by the maximum principle, $\sigma \equiv 0$ on $V \cap M^{\prime}$. Thus $V \cap M^{\prime} \subseteq \partial D$.

We have shown that, for each $p \in M$, there exists a neighborhood $V$ of $p$ so that $V \cap M^{\prime} \subseteq \partial D$. It follows that there is a neighborhood $U \subseteq W$ of $M$ so that $U \cap M^{\prime} \subseteq \partial D$.

REMARK. If $A(D):=H(D) \cap C(\bar{D})$, then it is easy to see that the assumption that $U \cap M^{\prime} \subseteq \partial D$ for a neighborhood $U$ of $M$ implies that each element of $A(D) \mid M$ is real-analytic. In fact, fixing $f \in A(D)$ and $q \in U \cap M^{\prime}$, we get that $f$ is locally near $q$ the uniform limit on $M^{\prime}$ of holomorphic functions by arguing as for $\sigma$ in step 3 above. It follows that $f \mid M$ is real-analytic.

3. Example of quasi-analyticity in the boundary. For the example we choose two nonnegative even functions $\phi$ and $\chi$ in $C^{\infty}(\mathbf{R})$ so that

(a) each is strictly convex off its zero set;

(b) $\chi^{-1}(0)=[-2,2]$;

(c) $\phi^{-1}(0)=\{0\} ;$ and

(d) $\phi$ vanishes to infinite order at 0 .

From [6, Example 4.1] we recall the domain $D$, defined near $K:=[-2,2] \times\{0\}$ in $\mathrm{C}^{2}$, by

$$
D:=\left\{(z, w) ; u+\chi(x)+\phi(y)+v^{2}\left(1+\frac{1}{100}|z|^{2}\right)<0\right\} ;
$$

here we use the notation $z=x+i y, w=u+i v . \quad D$ is convex and strongly pseudoconvex off of $K$, and $K$ is an integral curve. We put $L:=[-1,1] \times\{0\}$ and

$$
I_{k}=I_{k}(\phi):=\int_{0}^{1} \phi^{\prime}(t) t^{-k} d t \quad \text { for } k \geq 1 .
$$

LEMMA 1. Given $f \in A^{\infty}(D)$ there exists $C>0$ so that

$$
\left\|\partial^{k} f / \partial x^{k}\right\|_{L} \leq C k ! I_{k} \quad \text { for } k \geq 1 .
$$

ProOF. Lemma 4.1 of [6] gives this estimate with $L$ replaced by $\{(0,0)\}$, and one only needs to check that the estimate holds uniformly on $L$. For the convenience of the reader, we sketch the proof. If $k \geq 1$ then

$$
\frac{\partial^{k} f}{\partial x^{k}}(a, 0)=-\int_{0}^{1} \frac{d}{d t}\left[\frac{\partial^{k} f}{\partial x^{k}}(a,-\phi(t))\right] d t+\frac{\partial^{k} f}{\partial x^{k}}(a,-\phi(1))
$$


whenever $-1 \leq a \leq 1$. The integrand is bounded above by $k !\|\partial f / \partial w\| \bar{D} \phi^{\prime}(t) t^{-k}$ because of the Cauchy estimates for $\partial f / \partial w$ on discs in $\bar{D}$ of the form

$$
\{z ;|z-a| \leq t\} \times\{-\phi(t)\}
$$

the second term is similarly bounded above by $k !\|f\|_{\bar{D}}$. This gives the desired estimate.

The lemma shows that we can get gond regularity for $A^{\infty}(D) \mid L$ by choosing $\phi$ so that $I_{k}(\phi)$ grows slowly with $k$. The proof of the main theorem shows that we cannot choose $\phi$ so that, for some $C_{1}>0$,

$$
I_{k}(\phi) \leq C_{1}^{k} \text { for } k \geq 1 .
$$

Here is a more direct proof of this: Put

$$
\psi(t):= \begin{cases}0 & \text { if } t<1 \\ \phi^{\prime}(1 / t) & \text { if } t \geq 1\end{cases}
$$

The holomorphic Fourier transform $F$ of $\psi$ defined by

$$
F(z):=\int_{-\infty}^{\infty} \psi(t) e^{i t z} d t \quad(z \in \mathbf{C})
$$

would, if $(*)$ held, be an entire function of exponential type (a simple estimate); by the Paley-Wiener Theorem, $F$ would be the Fourier transform of a function with compact support, so $\psi$ would have compact support. Thus $(*)$ implies $\phi \equiv 0$ near 0 , contradicting (c) above. In the following lemma we indicate one possible construction of a $\phi$ whose growth rate approximates $(*)$.

LEMMA 2. Suppose $\left\{a_{k}\right\}$ is an unbounded increasing sequence with $a_{1} \geq 1$. Then there exists a function $\phi$ of the required form with

$$
I_{k}(\phi) \leq a_{k}^{k} \quad \text { for } k \geq 1 .
$$

Proof. Fix $\lambda \in C^{\infty}(\mathbf{R})$ so that $0 \leq \lambda \leq 1, \lambda(t) \equiv 0$ if $t \leq 1$, and $\lambda(t) \equiv 1$ if $t \geq 2$. If $j \geq 1$, let $c_{j}:=\max \left\{\left\|a_{j}^{k} \lambda^{(k)}\left(\bar{a}_{j} t\right)\right\|_{\mathbf{R}} ; 0 \leq k \leq j\right\}$; then $1 \leq c_{j}<\infty$. We define

$$
\psi(t):=\sum_{j=1}^{\infty} \lambda\left(a_{j} t\right) t^{j} /\left(c_{j} j^{j}\right) \quad \text { for } t \geq 0 .
$$

Then $\psi$ is infinitely differentiable, and $\psi>0$ if $t>0$. A rather crude estimate gives that, for $k \geq 2$,

$$
\begin{aligned}
\int_{0}^{1} \psi(t) t^{-k} d t & =\sum_{j=1}^{\infty} \int_{1 / a_{j}}^{1} \lambda\left(a_{j} t\right) t^{j-k} /\left(c_{j} j^{j}\right) d t \\
& \leq(k-1) a_{k}^{k}+1 .
\end{aligned}
$$

If we choose $\phi$ to be even and satisfy $\phi(0)=\phi^{\prime}(0)=0$ while $\phi^{\prime \prime}(t)=\psi(t)$ for $t \geq 0$, then integration by parts gives that, for some $C_{1}>0, I_{k}(\phi) \leq C_{1} a_{k}^{k}$ for $k \geq 1$. Dividing $\phi$ by $C_{1}$ gives the desired result.

EXAMPLE. Let $a_{k}=\log k$ for $k \geq 3$, and let $\phi$ be the corresponding function given in Lemma 2. By Lemma 1 , if $f \in A^{\infty}(D)$, then there exists $C>0$ so that

$$
\left\|\partial^{k} f / \partial x^{k}\right\|_{L} \leq C(k \log k)^{k} \quad \text { for } k \geq 3
$$


Since $\sum 1 /(k \log k)=\infty$, the Denjoy-Carleman Theorem (e.g., [4, Chapter IV, pp. $101 \mathrm{ff}$.]) implies that $A^{\infty}(D) \mid L$ is quasi-analytic. We remark that with the choice $\chi(2+t)=\phi(t)$ (for $t \geq 0$ ) it is straightforward to check that $A^{\infty}(D) \mid K$ is quasi-analytic.

The above example gives a result about peak sets for $A^{\infty}(D)$. Recall that a closed set $E$ in $\partial D$ is a peak set for $A^{\infty}(D)$ if there exists a function $g \in A^{\infty}(D)$ with $g=0$ on $E$ while $\operatorname{Re} g>0$ on $\bar{D} \backslash E$. $K$ is a peak set for $A^{\infty}(D)($ take $g=-w)$, but no subset $E$ of $(-1,1) \times\{0\}$ is a peak set for $A^{\infty}(D)$. In fact, if such a set $E$ were a peak set with corresponding function $g$, the function $f=\exp (-1 / \sqrt{g}) \in A^{\infty}(D)$ would vanish to infinite order on $E$. By the quasi-analyticity of $A^{\infty}(D) \mid L, f \equiv 0$ on $L$, so $E \supseteq L$, a contradiction. (A different proof of a related fact about peak sets in $K$ is given in [5, Example 1.1].)

REMARK. In contrast to the above phenomena, $A(D)$ gains no regularity upon restriction to $K$ in the above examples. More precisely, $A(D) \mid K=C(K)$, i.e., $K$ is an interpolation set for $A(D)$. The proof is as follows: Since $K$ is a peak set, a well-known result from the theory of uniform algebras (e.g., [1, Corollary 2.4 .3 , p. 104]) implies that $A(D) \mid K$ is uniformly closed in $C(K)$. In addition, the Stone-Weierstrass Theorem implies that holomorphic polynomials are dense in $C(K)$. Thus $A(D) \mid K=C(K)$.

\section{REFERENCES}

1. A Browder, Introduction to function algebras, Benjamin, New York, 1969.

2. K. Diederich and J. E. Fornaess, Pseudoconvex domains: bounded strictly plurisubharmonic exhaustion functions, Invent. Math. 39 (1977), 129-141. MR 55 \#10728.

3. M. Hakim and N. Sibony, Spectre de $A(\bar{\Omega})$ pour des domaines bornés faiblement pseudoconvexes réguliers, J. Funct. Anal. 37 (1980), 127-135. MR 81 \#46072.

4. S. Mandelbrojt, Séries adhérentes, regularisation des suites, applications, Gauthier-Villars, Paris, 1952.

5. A. Noell, Peak points in boundaries not of finite type, Pacific J. Math. (to appear).

6. Interpolation in weakly pseudoconvex domains in $\mathbf{C}^{2}$, Math. Ann. (to appear).

7. W. Rudin, Functional analysis, McGraw-Hill, New York, 1973.

8. N. Sibony, Prolongement analytique des fonctions holomorphes bornées, C. R. Acad. Sci. Paris Ser. A 275 (1972), 973-976. MR 47 \#7062.

9. E. Stein, Singular integrals and estimates for the Cauchy-Riemann equations, Bull. Amer. Math. Soc. (N.S.) 79 (1973), 440-445. MR 47 \#3851.

10. R. O. Wells, Holomorphic approximation on real-analytic submanifolds of a complex manifold, Proc. Amer. Math. Soc. 17 (1966), 1272-1275. MR 34 \#832.

Department of MATHEMAtics 253-37, CAlifornia Institute of TeChNology, PASADENA, CALIFORNIA 91125 